\title{
Benefit of radiofrequency ablation after widespread endoscopic resection of neoplastic Barrett's esophagus in daily practice
}

\author{
Sébastien Godat ${ }^{a *}$, Mariola Marx ${ }^{a *}$, Fabrice Caillol ${ }^{b}$, Maxime Robert ${ }^{a}$, Aurélie Autret ${ }^{c}$, Erwan Bories ${ }^{b}$, \\ Christian Pesenti ${ }^{b}$, Jean Philippe Ratone ${ }^{b}$, Alain Schoepfera ${ }^{a}$, Flora Poizat ${ }^{d}$, Marc Giovannini ${ }^{b}$
}

CHUV, Lausanne, Switzerland; Paoli-Calmettes Institute, Marseille, France

\section{Abstract}

Division of ${ }^{\mathrm{a}}$ Gastroenterology and Hepatology, CHUV, Lausanne, Switzerland (Sébastien Godat, Mariola Marx, Maxime Robert, Alain Schoepfer); 'Gastroenterology, Paoli-Calmettes Institute, Marseille, France (Fabrice Caillol, Erwan Bories, Jean Philippe Ratone, Marc Giovannini); 'Biostatistics, Paoli-Calmettes Institute, Marseille, France (Aurélie Autret); ${ }^{\mathrm{D} D i v i s i o n}$ of Pathology, Paoli-Calmettes Institute, Marseille, France (Flora Poizat)

Conflict of Interest: None

${ }^{\star}$ Equal contributions

Correspondence to: Dr Sébastien Godat, MD, Division of Gastroenterology and Hepatology, Centre Hospitalier Universitaire Vaudois (CHUV) University of Lausanne, Rue du Bugnon 44, CH-1011 Lausanne, Switzerland, e-mail: Sebastien.Godat@chuv.ch

Received 9 October 2020; accepted 2 March 2021; published online 6 December 2021

DOI: https://doi.org/10.20524/aog.2021.0685

\section{Introduction}

Barrett's esophagus (BE) is the replacement of squamous mucosa by specialized intestinal metaplasia and represents a risk factor for the development of esophageal cancer, according to the sequence: metaplasia - low-grade dysplasia (LGD) high-grade dysplasia (HGD) - esophageal adenocarcinoma (EAC) [1]. Nowadays, the perioperative morbidity, as well as the mortality risk associated with surgical esophageal resection, still remains non-negligible, even for procedures performed by experienced teams [2-5]. In contrast, the risk of lymph node metastasis in adenocarcinoma confined to the mucosal or superficial submucosal layer (sm1) with lowrisk features is $<1.5 \%$ [6-10]. Based on these considerations, HGD and superficial EAC in BE are now well-established indications for endotherapy (ET) $[1,11]$, allowing preservation 
of the esophageal anatomy with an excellent safety profile and a curative efficiency up to $98 \%[12,13]$. The associated morbidity is lower than that of surgery, with a maximum morbidity rate of $19 \%$ and a mortality rate of $0 \%$, while most studies on the subject found even lower morbidity rates $[14,15]$.

Long-term data have confirmed that complete eradication of non-dysplastic BE after treatment of neoplastic lesions is crucial to reduce the risk of tumor recurrence and metachronous lesions $[15,16]$. However, for a long time there was disagreement as to whether endoscopic resection (ER) or thermal ablation was the preferred eradication strategy for remaining $\mathrm{BE}$, balancing the advantage of histological reliability against potential adverse events, such as bleeding, perforation or post-interventional stenosis [17].

The currently recommended management is to combine endoscopic mucosal resection (EMR) or endoscopic submucosal dissection (ESD) of all visible or irregular lesions, complemented by radiofrequency ablation (RFA) of all flat remnant Barrett's tissue [18]. This combination therapy provides the advantage of a histopathologically confirmed diagnosis and has shown equivalent or better results compared to RFA alone for neoplastic $\mathrm{BE}$, with a low recurrence rate $<10 \%$ for neoplastic and minor non-dysplastic BE [18-21].

Until 2010, the policy in our endoscopy unit was to perform a concomitant complete macroscopic resection of metaplastic BE in cases of ET for neoplastic lesions. From 2010, RFA was used as adjuvant therapy in the management of Barrett's with HGD/intramucosal carcinoma (IMC), to eradicate residual intestinal metaplasia (IM) after widespread ER [14,22,23]. The principal objective of our study was to analyze the benefit and safety of complementary RFA in current practice compared to widespread ER alone.

\section{Patients and methods}

We carried out a retrospective observational study in a single tertiary referral endoscopy center, monitored and approved by the Institutional Review Board. From computerized and retrospectively collected data of our endoscopy and hospital database (4D program ${ }^{\circledast}$ ), we retrieved all patients who underwent ET for neoplastic or dysplastic BE between 2006 and 2013. In the absence of recent data, we obtained supplementary parameters by contacting the patients' general practitioners or referring gastroenterologists. The various clinical, endoscopic, histopathological and technical characteristics were manually collected in a computer database and finally analyzed by an affiliated biostatistician. We recorded patients' characteristics, initial macroscopic Barrett's length using the Prague classification, detailed description of ET sessions, histological diagnosis based on preoperative biopsies and on the specimen after resection, occurrence and treatment of adverse events, delay between initial endoscopic treatment and relapse, the rate of cancerous progression, as well as the rate of salvage therapy, such as chemoradiotherapy or surgery.

Inclusion criteria were defined as the presence of HGD or EAC on pre-resection biopsies or on histological specimen after
ER. For early esophageal carcinoma we required tumor-free resection margins with a maximum depth infiltration of $\mathrm{m} 3$ according to the Japanese Society of Esophageal Disease [24]. Lesions infiltrating the submucosa (T1b) were excluded in accordance with our institution's policy.

ER, for both visible lesions and the majority of surrounding $\mathrm{BE}$ tissue, was done by EMR or ESD, depending on the practitioner's choice, given the macroscopic presentation and histopathological evaluation. EMR procedures were accomplished using a snare or the multiband ligation Duette device (Cook Medical) after submucosal injection of saline solution colored by indigo carmine. To perform ESD we used the Dual Knife (Olympus) after outline marking and submucosal injection of saline solution colored by indigo carmine. Both EMR and ESD were preferentially performed semi-circumferentially, except for circumferential resection in some cases of short BE. If diminutive residual $\mathrm{BE}$ islands were found in the combination treatment group, we erased the residual $\mathrm{BE}$ area using adjuvant RFA (Covidien ${ }^{\circledR}$ ). If symptomatic esophageal stricture occurred, we performed endoscopic balloon dilation (Hercules ${ }^{\circledast}$ ) in as many sessions as were necessary to resolve dysphagia and to permit new ER. All patients were on a standard dose of a proton pump inhibitor b.i.d. during and after completion of ET. ET sessions were carried out every 4-12 weeks.

Endoscopic monitoring was performed every 6 months after treatment, with targeted biopsies taken if there were suspicious lesions. Systematic biopsies were performed on the Z-line if no suspicious lesion was visualized. Macroscopic eradication of metaplastic BE was considered as the end of treatment, while histological confirmation was not mandatory. In contrast, eradication of HGD/EAC had to be systematically confirmed by histopathological analysis. Once ET was completed, patients were recommended to continue biannual endoscopic and histological follow up.

We analyzed the outcomes of 2 different subgroups: patients in group 1 were treated by widespread ER alone, including the visible lesion and the surrounding $\mathrm{BE}$, whereas patients in group 2 had a multimodal treatment strategy with widespread ER followed by RFA. Treatment success was defined as the absence of HGD/IMC during endoscopic and histological control after at least 6 months of follow up. ET has been considered as failure if eradication of the neoplastic part of BE required more than 3 sessions, based on the histopathological analysis of the resected mucosa. Additional treatment sessions for complete eradication of non-dysplastic BE have been accepted. We defined relapse as the occurrence of HGD/IMC in any localization within the BE mucosa after initial treatment success, which means after one negative endoscopic control and not less than 6 months of follow up.

\section{Results}

Between 2006 and 2013, 92 patients underwent ET for neoplastic BE. Since 3 patients with an infiltration depth of $\mathrm{sm} 1 / \mathrm{sm} 2$ (T1b) were excluded, our results are based on 89 patients. Until 2010, a total of 31 patients underwent ER; 13 
of them underwent additional treatment by RFA when it was available after 2010. In the period from 2010-2013, 37 patients were treated by ER alone, while in 21 patients we performed a combination of ER followed by RFA (Fig. 1). Mean followup time for all patients was 23.6 months (range 6-84, 95\% confidence interval [CI] 19.6-28.1).

\section{Patients' baseline characteristics}

Fifty-five patients with a mean age of 68 years, 48 men and 7 women, were treated by ER alone (Table 1). Their mean BE length was C2.7 cm (range 0-14 cm, 95\%CI 1.7-3.7), M4.5 cm (range 1-17 cm, 95\%CI 3.7-5.2). At initial macroscopic evaluation, visible lesions presented a mean size of $16.5 \mathrm{~mm}$ (range $8-30 \mathrm{~mm}$ ) and were described as nodular in 17 cases (30.9\%, Paris 0-Is/0-IIa), as flat in 36 (65.4\%, Paris 0-IIb) and depressed in 2 cases (3.6\%, Paris 0-IIc). Histological preoperative analysis revealed HGD in 53 (96\%) samples (Table 2). In contrast, resection specimens confirmed HGD in 13 (23.6\%), but LGD in 16 (29.1\%) and intestinal metaplasia in $7(12.7 \%)$ cases. IMC was found in $4(7.3 \%)$ patients classified as pT1m2N0M0, and in $15(27.3 \%)$ patients classified as pT1m3N0M0. Two patients with LGD on preoperative samples, but endoscopically suspect lesions, showed HGD on a surgical specimen. The total mismatch rate between presumptive and final histological diagnosis was 45.5\% (Table 2).

Treatment combining ER and RFA was performed in 34 patients, 31 men and 3 women, with a mean age of 67 years and a mean BE Prague classification of C4.4 cm (range 0-13 cm, 95\%CI 3.3-5.8), M6.6 cm (range 2-13 cm, 95\%CI 5.7-7.4). Visible lesions were endoscopically characterized as nodular

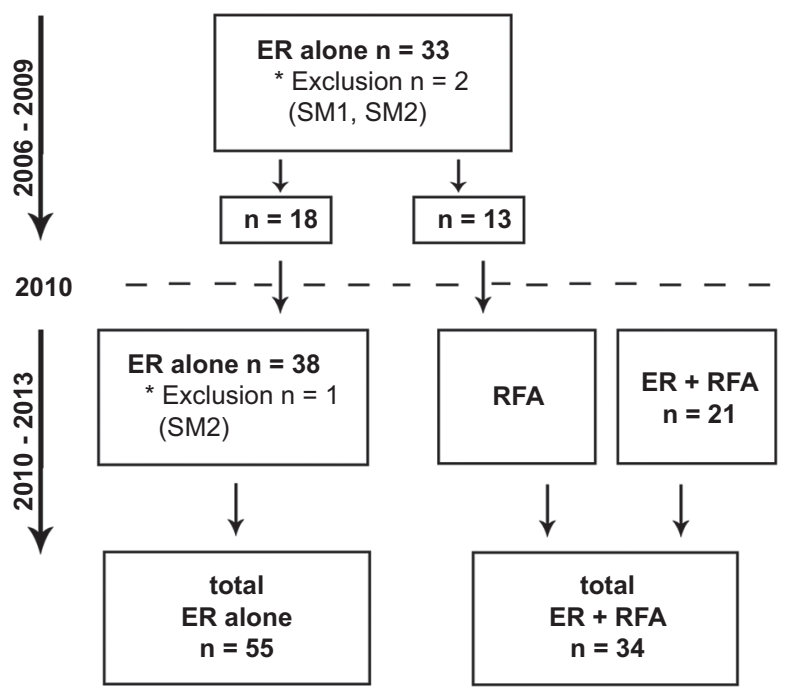

Figure 1 Flowchart showing endoscopic treatment of included patients: Until 2010 patients have exclusively been treated by endoscopic resection (ER) for neoplastic Barrett's esophagus (BE) and eradication of remnant non-dysplastic BE. Complementary treatment for nondysplastic BE by radiofrequency ablation (RFA) was introduced later and mainly used in long $\mathrm{BE}>5 \mathrm{~cm}$ after resection of neoplastic $\mathrm{BE}$ in 8 patients (23.5\%, Paris 0 -Is/0-IIa), as flat in $23(67.6 \%$, Paris 0 -IIb) and as depressed in 3 patients (8.8\%, Paris 0-IIc). Their mean size was $13.4 \mathrm{~mm}$ (range $10-25 \mathrm{~mm}$ ). Preoperative histological analysis was positive for HGD in 32 (94.1\%) patients and for LGD in 2 (5.9\%) patients. Final histological diagnosis after resection revealed HGD in 13 (38.2\%) and LGD in $5(14.7 \%)$ patients, with metaplasia and normal stratified squamous epithelium in $1(2.9 \%)$ case. IMC was found in 14 patients, $5(14.7 \%)$ cases classified as pT1m2N0M0 and $9(26.5 \%)$ cases as pT1m3N0M0. The mismatch rate between preoperative and definitive histological diagnosis was $26.5 \%$.

\section{Treatment}

In patients treated exclusively by ER (group 1), EMR procedures were performed in a total of $53(96.4 \%)$ patients, using a snare technique in $8(14.5 \%)$ patients, multiband ligation with the Duette ${ }^{\circledast}$ device in $30(54.5 \%)$ patients and a dual-channel scope in $15(27.3 \%)$ patients (Table 3). Two (3.6\%) patients underwent ESD.

EMR in group 2 (ER+RFA) was carried out in 31 (91.2\%) patients. Multiband ligation with the Duette ${ }^{\circledast}$ device was used in 18 (52.9\%) subjects, a snare technique in 7 (20.6\%) and resection by the dual-channel scope in $6(17.6 \%)$. ESD was performed in $3(8.8 \%)$ patients. All patients in this group underwent combination therapy. Focal residual $\mathrm{BE}$ areas were treated in all 34 patients by RFA with the Halo $90^{\circ}$ device in a mean number of 1.6 (range 1-4) sessions. Six of them had additional circumferential RFA using the Halo $360^{\circ}$ device in only one session per patient. There was no significant difference in mean treatment duration, requiring 1.8 treatment sessions (range 1-4) over 4.8 (range 2-12) months in group 1 and 2 treatment sessions (range 1-7) over 5.1 (range 2-13) months in group 2.

\section{Adverse events and safety outcomes}

Complications occurred in 8 of the $34(23.5 \%)$ patients who received combination therapy, compared with 9 of the $55(16.4 \%)$ patients who had ER alone (Table 4$)$. There were no deaths related to ET in either group. Stricture development was observed in 8 patients who had ER alone (14.5\%). Three of them developed stenosis after one circumferential mucosal resection, 1 patient after a $75 \%$ resection, 2 patients after the second hemi-circumferential resection, while in 2 patients the resected surface was not exactly quantified. In 6 patients treated by combination therapy, esophageal stenosis occurred after widespread ER and not after RFA sessions (17.6\%): in 2 patients after the first hemi-circumferential resection, in 2 patients after the second hemi-circumferential resection and in 2 patients after large resection (area not quantified). Of the 6 patients treated using the Halo $360^{\circ}$ device, none showed post-interventional stenosis. Subsequent esophageal stenosis required endoscopic dilatation in a mean number of 2.1 and 2.3 sessions for groups 1 and 2, respectively. There were no stricture recurrences on successive endoscopies. 
Table 1 Baseline characteristics of included patients

\begin{tabular}{|c|c|c|}
\hline Characteristics & Group 1: ER & Group 2: ER+RFA \\
\hline Number of patients, $\mathrm{n}$ & 55 & 34 \\
\hline Sex, $n[\%]$ & $48+7$ & $31+3$ \\
\hline Age, mean [range] in years & $68[48-86]$ & $67[40-88]$ \\
\hline ASA score, mean & 2.1 & 2.1 \\
\hline Initial Prague classification, mean in $\mathrm{cm}$ [range; CI] & $\begin{array}{l}\text { C2.7 }[0-14 ; 1.7-3.7] \\
\text { M4.5 }[1-17 ; 3.7-5.2]\end{array}$ & $\begin{array}{l}\text { C4.4 }[0-13 ; 3.3-5.8] \\
\text { M6.6 }[2-13,5.7-7.4]\end{array}$ \\
\hline $\begin{array}{l}\text { Initial macroscopic presentation, } \mathrm{n} \text { [\%] } \\
\text { Paris classification } \\
\text { 0-Is/0-IIa } \\
\text { 0-IIb } \\
\text { 0-IIc }\end{array}$ & $\begin{array}{c}17[30.9] \\
36[65.4] \\
2[3.6]\end{array}$ & $\begin{array}{c}8[23.5] \\
23[67.6] \\
3[8.8]\end{array}$ \\
\hline $\begin{array}{l}\text { Initial size of cancerous nodule, mean, mm } \\
\text { [range] } \\
\quad \leq 20 \mathrm{~mm}, n[\%] \\
\quad>20 \mathrm{~mm}, n[\%]\end{array}$ & $\begin{array}{c}16.5[8-30] \\
14[25.4] \\
4[7.3]\end{array}$ & $\begin{array}{l}13.4[10-25] \\
\quad 9[26.4] \\
\quad 2[5.9]\end{array}$ \\
\hline
\end{tabular}

ER, endoscopic resection; RFA, radiofrequency ablation; ASA, American Society of Anesthesiologists; $\hat{0}$, male; , female, Prague classification, C circumferential and $M$ maximum length of Barrett's esophagus; $C I$, confidence interval

Table 2 Preoperative and final histological diagnosis

\begin{tabular}{|c|c|c|c|c|}
\hline Groups & Preoperative diagnosis & $\mathrm{n}[\%]$ & Final histological diagnosis on surgical specimen & $\mathrm{n}[\%]$ \\
\hline $\begin{array}{l}\text { Group } 1 \\
\text { (ER alone) }\end{array}$ & $\begin{array}{l}\text { LGD } \\
\text { HGD }\end{array}$ & $\begin{array}{c}2[3.6] \\
53[96.4]\end{array}$ & $\begin{array}{l}\text { HGD } \\
\text { Normal squamous epithelium } \\
\text { Metaplasia } \\
\text { LGD } \\
\text { HGD/pTisN0M0 } \\
\text { Intramucosal carcinoma } \\
\text { pT1m2N0M0 } \\
\text { pT1m3N0M0 }\end{array}$ & $\begin{array}{c}2[3.6] \\
0[0] \\
7[12.7] \\
16[29.1] \\
11[20] \\
\\
4[7.3] \\
15[27.3]\end{array}$ \\
\hline $\begin{array}{l}\text { Group } 2 \\
\text { (ER+RFA) }\end{array}$ & $\begin{array}{l}\text { LGD } \\
\text { HGD }\end{array}$ & $\begin{array}{c}2[5.9] \\
32[94.1]\end{array}$ & $\begin{array}{l}\text { HGD } \\
\text { Normal squamous epithelium } \\
\text { Metaplasia } \\
\text { LGD } \\
\text { HGD/pTisN0M0 } \\
\text { Intramucosal carcinoma } \\
\text { pT1m2N0M0 } \\
\text { pT1m3N0M0 }\end{array}$ & $\begin{array}{r}2[5.9] \\
1[2.9] \\
1[2.9] \\
5[14.7] \\
11[32.4] \\
\\
5[14.7] \\
9[26.5]\end{array}$ \\
\hline
\end{tabular}

ER, endoscopic resection; RFA, radiofrequency ablation; $L G D$, low-grade dysplasia; $H G D$, high-grade dysplasia

Perforation occurred in one patient treated by EMR alone (1.8\%), whereas there was no perforation in patients with combination therapy. The mucosal defect was successfully closed by temporary placement of an esophageal stent. Of the patients who underwent EMR followed by RFA, 2 (5.9\%) suffered perioperative bleeding, managed by endoscopic hemostasis. In summary, complication rates did not differ statistically between the 2 treatment groups $(\mathrm{P}=0.58)$ and all complications could be treated conservatively or endoscopically.

\section{Efficacy outcomes}

Complete eradication for intestinal metaplasia was achieved in 20 of 34 patients who had RFA after ER (58.8\%), compared to 48 of the 55 patients treated by ER alone (87.3\%). In contrast, the complete eradication rate for neoplastic BE was equivalent for both groups, as set out in Table 5. Thus, treatment success by ER alone (group 1) was confirmed in all 32 patients. In patients getting combination therapy by ER and RFA (group 2) treatment failure was observed in only one patient, while complete eradication was successful in 26 of 27 (96.3\%, $\mathrm{P}=0.93)$ patients.

We observed no significant difference between the 2 groups in the recurrence rate of HGD or neoplasia, with a relapse rate of $9.1 \%(5 / 55)$ in patients treated by ER alone and a relapse rate of $14.7 \%(5 / 34)$ in patients treated by ER and RFA $(\mathrm{P}=0.42)$ (Table 6). The mean interval between complete eradication and relapse was 16.6 months in group 1 (ER alone) and 18.2 months in group $2(\mathrm{ER}+\mathrm{RFA})$. We observed the latest relapse after 33 months in group 1 and after 28 months in group 2. Recurrent 
Table 3 Endoscopic treatment strategy

\begin{tabular}{lcc} 
Endotherapy & Group 1: ER & Group 2: ER+RFA \\
\hline Endoscopic resection, $\mathrm{n}$ [\%] & & \\
EMR & $8[14.5]$ & $7[20.6]$ \\
$\quad$ Snare & $15[27.3]$ & $6[17.6]$ \\
$\quad$ Dual channel & $30[54.5]$ & $18[52.9]$ \\
$\quad$ Multiband ligation EMR, Duette & $2[3.6]$ & $3[8.8]$ \\
ESD & $1.8[1-4]$ & $1.97[1-7]$ \\
Number of ER sessions, mean [range] & NA & $34[100]$ \\
RFA, n (\%) & & $6[17.6]$ \\
Halo $90^{\circ}$ & NA & $1.62[1-4]$ \\
Halo $360^{\circ}$ & & 1 session per patient \\
Number of RFA sessions, mean [range] & $4.8[2-12]$ & $5.1[2-13]$ \\
\hline Halo $360^{\circ}$ & \\
Duration of treatment, months, mean [range]
\end{tabular}

ER, endoscopic resection; RFA, radiofrequency ablation; EMR, endoscopic mucosal resection; ESD, endoscopic submucosal dissection

\section{Table 4 Adverse events}

\begin{tabular}{lcc}
\hline Adverse events & Group 1: ER & $\begin{array}{c}\text { Group 2: } \\
\text { ER+RFA }\end{array}$ \\
\hline Perioperative bleeding, $\mathrm{n}[\%]$ & 0 & $2[5.9]$ \\
Stricture development, $\mathrm{n}[\%]$ & $8[14.5]$ & $6[17.6]$ \\
Perforation, $\mathrm{n}$ [\%] & $1[1.8]$ & 0 \\
Death, $\mathrm{n}$ [\%] & 0 & 0 \\
Number of endoscopic dilation & $2.1[2-3]$ & $2.3[2-3]$ \\
sessions, mean [range] & & \\
Total rate of adverse events, [\%] & 16.4 & 23.5 \\
\hline
\end{tabular}

ER, endoscopic resection; $R F A$, radiofrequency ablation

Table 5 Treatment success after ER alone versus ER+RFA

\begin{tabular}{lcc}
\hline Complete eradication & $\begin{array}{c}\text { Group 1: } \\
\text { ER }\end{array}$ & $\begin{array}{c}\text { Group 2: } \\
\text { ER+RFA }\end{array}$ \\
\hline Metaplasia, n [\%] & $48 / 55[87.3]$ & $20 / 34[58.8]$ \\
LGD, n [\%] & $15 / 16[93.8]$ & $5 / 5[100]$ \\
$\begin{array}{l}\text { HGD/pTisN0M0 or } \\
\text { intramucosal carcinoma, n [\%] }\end{array}$ & $13 / 13[100]$ & $12 / 13[92.3]$ \\
$\begin{array}{l}\text { IMC, n [\%] } \\
\text { pT1m2N0M0 }\end{array}$ & $4 / 4[100]$ & $5 / 5[100]$ \\
pT1m3N0M0 & $15 / 15[100]$ & $9 / 9[100]$ \\
$\begin{array}{l}\text { Total eradication rate for HGD } \\
\text { or IMC, \% }\end{array}$ & 100 & 96.3 \\
\hline
\end{tabular}

ER, endoscopic resection; $R F A$, radiofrequency ablation; $L G D$, low-grade dysplasia; HGD, high-grade dysplasia; is, in situ; IMC, intramucosal carcinoma

disease was first treated endoscopically. Complementary surgical management was needed in $5(9.1 \%)$ patients treated by ER alone and in 1 (2.9\%) patient treated by ER and RFA. Two (3.6\%) patients in group 1 and 1 (2.9\%) patient in group 2 had complementary chemoradiotherapy. There was no death related to the disease. One patient presented metastatic progression, but died finally in an accident 77 months after the initial treatment.
Table 6 Long-term outcome after endotherapy

\begin{tabular}{|c|c|c|}
\hline Relapse and follow up & $\begin{array}{l}\text { Group 1: } \\
\text { ER }\end{array}$ & $\begin{array}{l}\text { Group 2: } \\
\text { ER+RFA }\end{array}$ \\
\hline Relapse rate, $\mathrm{n}[\%]$ & $5 / 55[9.1]$ & $5 / 34[14.7]$ \\
\hline $\begin{array}{l}\text { Delay for relapse, mean [range] in } \\
\text { months }\end{array}$ & $16.6[6-33]$ & $18.2[7-28]$ \\
\hline Metastasis progression, $\mathrm{n}[\%]$ & $1[1.8]$ & 0 \\
\hline Deaths related to the disease, $\mathrm{n}$ [\%] & 0 & 0 \\
\hline $\begin{array}{l}\text { Deaths unrelated to the disease, } \mathrm{n}[\%] \\
\text { Accidental death } \\
\text { Concomitant pancreatic cancer } \\
\text { Sepsis post esophagectomy for } \\
\text { relapse }\end{array}$ & $\begin{array}{c}1[1.8] \\
0 \\
1[1.8]\end{array}$ & $\begin{array}{c}0 \\
1[2.9] \\
0\end{array}$ \\
\hline $\begin{array}{l}\text { Complementary surgical } \\
\text { management for relapse, } \mathrm{n}(\%)\end{array}$ & 5 [9.1] & 1 [2.9] \\
\hline $\begin{array}{l}\text { Complementary chemoradiotherapy } \\
\text { for relapse, } \mathrm{n}(\%)\end{array}$ & $2[3.6]$ & $1[2.9]$ \\
\hline
\end{tabular}

\section{Discussion}

ET is the standard of care for HGD and early neoplastic lesions in $\mathrm{BE}$, and is associated with lower morbidity and mortality compared to surgery $[3,25]$. Recent studies provided good evidence for excellent long-term outcomes [12,26]. ER preserves the esophageal anatomy, while the histological analysis of the resected specimen allows the need for further surgical therapy to be defined, depending on tumor differentiation, infiltration depth and lymphovascular invasion.

Current guidelines recommend ER of every visible abnormality containing any degree of dysplasia or neoplasia, followed by complete eradication of all remaining Barrett's epithelium, preferably by RFA [27]. Widespread ER remains common practice in some endoscopy units, as is the case in our department, whereas adjuvant RFA is primarily reserved for eradication of long segment BE after ER of neoplastic lesions [12,22,23,28]. 
We report here on our experience in the management of neoplastic BE, based on a retrospective analysis of patients treated by ER alone vs. a combination of ER followed by RFA. RFA was introduced in our endoscopy unit in 2010. At the beginning, the French health insurance did not completely cover costs and RFA had to be employed with restrictions due to financial issues. Thus, our results are based on a heterogeneous treatment management, but reflect outcomes in current daily practice.

Patients' baseline characteristics were found to be similar in both groups, except for the circumferential and maximum extent of Barrett's mucosa, significantly longer in patients undergoing combination therapy than in patients treated by ER alone (mean Prague classification C4.4M6.6 vs C2.7M4.5, $\mathrm{P}<0.001)$. This trend towards longer $\mathrm{BE}$ observed in patients treated by ER+RFA (mainly after 2010), could be associated with an increase in the prevalence of gastroesophageal reflux disease during the last decades and is concordant with the growing incidence of EAC [29,30]. Furthermore, this difference might be in part explained by our department's policy, using adjuvant RFA for treatment of remnant $\mathrm{BE}>5 \mathrm{~cm}$ to reduce the risk of esophageal stricture formation, as RFA shows lower esophageal stricture rates compared to ER [13]. In contrast, large mucosal resection and resection of multiple lesions on the initial procedure are known risk factors for esophageal stricture formation [31]. Esophageal stenosis is a well described and challenging complication of ET, which might impede ongoing treatment by contraindicating complementary resection/ ablation. Furthermore, repetitive therapeutic dilations bear a non-negligible risk of perforation. There are similar results in the USA RFA Registry, analyzing data from 1263 patients with HGD or IMC, treated by EMR for nodular lesions before RFA in 406 (32\%) and by RFA only in 857 (68\%) patients in the case of non-nodular BE. While there were no differences concerning the efficacy of eradication or complications, the investigators found that the $\mathrm{BE}$ length of patients treated by EMR before RFA was shorter than that of patients treated with RFA only (4.6 vs. $5.4 \mathrm{~cm}, \mathrm{P}<0.001$ ) [32].

Despite the discrepancy in BE length, there was no significant difference between the groups concerning the complete eradication rate of HGD or IMC (100\% vs. $96.3 \%$, $\mathrm{P}=0.93$ ), suggesting that combination treatment allows larger Barrett's areas to be treated with no decrease in efficacy. In addition, combination treatment did not increase the rate of procedure-related complications. The occurrence of esophageal strictures has mainly been observed after ER, even in patients treated by combination therapy. However, dilation of the stenosis was always successful and never hampered later treatment by ER or RFA.

In contrast, treatment success for eradication of intestinal metaplasia was significantly higher in patients treated by widespread resection alone, compared to the multimodal endoscopic treatment group $(87.3 \%$ vs. $58.8 \%, \mathrm{P}=0.002)$, probably reflecting a suboptimal implementation of RFA due to cost issues. Indeed, RFA was performed in a mean number of 1.6 sessions (range 1-4), until eradication of endoscopic visible BE. In contrast, prospective studies published during recent years performed a median of 3-4 sessions, with histological confirmation of non-dysplastic BE eradication [18]. Though we required histologically confirmed eradication of neoplastic BE, eradication of non-dysplastic BE was based on macroscopic evaluation. The decision in favor of macroscopic IM eradication-and thus against performance of RFA without visible lesions-was influenced by the risk/ benefit ratio, considering the slightly elevated risk of relapse acceptable to minimize the risk of repeated interventions and general anesthesia.

The above-mentioned difference in IM eradication rate may also explain in part the sustainability of the treatment response: thus, we found a higher, though not statistically significant, relapse rate of $\mathrm{HGD} / \mathrm{EAC}$ in patients treated by multimodal therapy compared to ER alone $(14.7 \%$ vs. 9.1\%, $\mathrm{P}=0.42)$. Because of a lack of awareness at the beginning of this study, we did not report the localization of relapse, and we assume that in some cases "relapse" may represent a metachronous lesion rather than a real local recurrence. The first good evidence suggesting a benefit from complete eradication of IM was published by Pech et al in 2008: the investigators detected significantly less metachronous neoplasia in patients receiving ablation therapy compared to patients without complementary treatment (16.5 vs. $29.9 \%$ ) [15]. These data correlate with our previous published study, showing a relapse rate of $3.4 \%$ $(1 / 29$ patients) in case of complete eradication of metaplastic $\mathrm{BE}$, but $31 \%(9 / 29)$ in case of incomplete eradication [16]. However, the present study included patients from 20062013 and the importance of complete eradication of IM only became evident during the later years and was not so obvious at the beginning of our study. Thus, our results underline once again the importance of complete eradication of remaining $\mathrm{BE}$ after treatment of HGD/EAC to decrease the risk of neoplasia relapse.

The management of relapse in patients treated by widespread resection alone required more complementary surgical and chemoradiotherapeutic treatment sessions than in the ER+RFA treatment group. Complementary surgical management was needed in all 5 relapsed patients $(9.1 \%)$ treated by ER alone. Only 1 patient presenting relapse in the ER+RFA group underwent surgery. Two patients in group 1 (3.6\%) and 1 patient in group $2(2.9 \%)$ had complementary chemoradiotherapy. Though recurrent lesions were first treated by endoscopic treatment, most of the reported cases occurred before the implementation of ESD in our endoscopy unit: thus, management of complicated relapse was technically difficult, and this may in part explain the substantial use of secondary surgery [33].

The key point of our present study is a significantly higher mismatch between the preoperative and final histological diagnosis in patients treated by ER alone than in patients treated by combination therapy ( 45.4 vs. $26.5 \%)$, suggesting that the greater the therapeutic resection, the more likely we are to get a reliable histological diagnosis. Preoperative biopsies of suspect lesions in BE are known to have poor reliability, and 3 patients with initial HGD were diagnosed with submucosal infiltration (T1b) after widespread resection. They were referred for further surgical management. Thus, complete 
resection continues to be the preferred procedure to obtain an accurate histological classification [28,34-36].

Histological staging by ER is crucial to determine a patient's optimal treatment strategy. According to the USA RFA Registry, the relapse rate was shown to be lower in case of ER of visible lesions preceding RFA, despite a comparable complete eradication rate of advanced dysplasia or IMC in both groups. Thus, $1.5 \%$ (5/331) of patients who had EMR before RFA progressed to invasive neoplasia, whereas $3.6 \%$ (24/663) of patients treated by RFA alone developed invasive adenocarcinoma $(\mathrm{P}=0.07)$. Among the latter 24 patients, 23 progressed from HGD [32]. These data suggest that, when RFA is used for treatment of non-nodular HGD, more advanced lesions might be missed and might progress to invasive adenocarcinoma.

In a retrospective study of 78 patients in 4 tertiary medical centers in the US, EMR prior to RFA as the primary treatment for biopsy-proven IMC significantly reduced the risk of treatment failure, observed in 4/6 patients treated by RFA alone vs. $13 / 72$ patients treated by ER+RFA (hazard ratio 0.15 , 95\%CI 0.05-0.48; $\mathrm{P}=0.001$ ) [37]. Similarly, in the UK patient registry, the rate of ER of visible suspicious lesions prior to RFA increased between 2008 and 2013 from $48 \%$ to $68 \%(\mathrm{P}=0.013)$. In the same time, the relapse rate decreased from $13 \%$ to $2 \%$, suggesting that ER improves the efficacy of endoscopic treatment of BE [17].

To conclude, the association of RFA with widespread ER does not increase the complication rate, but allows ET of significantly longer neoplastic BE with similar treatment success. However, the use of RFA needs careful consideration, given that it does not enable accurate histological staging. ER, in contrast, is the only method to obtain a reliable histological diagnosis.

\section{Summary Box}

\section{What is already known:}

- High-grade dysplasia (HGD) and intramucosal carcinoma (IMC) in Barrett's esophagus (BE) are well-established indications for endoscopic treatment

- Radiofrequency ablation (RFA) can be associated with endoscopic resection (ER) in case of flat or long-segment $\mathrm{BE}$ ablation

\section{What the new findings are:}

- The combination of ER and RFA can treat significantly longer BE with HGD/IMC than ER alone, with the same efficacy

- A multimodal treatment strategy (ER+RFA) does not increase the complication rate of endotherapy

- ER is mandatory to ensure a correct histological diagnosis of HGD and IMC

\section{References}

1. Shaheen NJ, Falk GW, Iyer PG, Gerson LB; American College of Gastroenterology. ACG Clinical Guideline: Diagnosis and management of Barrett's esophagus. Am J Gastroenterol 2016;111:30-50.

2. Awad ZT, Abbas S, Puri R, Dalton B, Chesire DJ. Minimally invasive Ivor Lewis esophagectomy (MILE): technique and outcomes of 100 consecutive cases. Surg Endosc 2020;34:3243-3255.

3. Bennett C, Vakil N, Bergman J, et al. Consensus statements for management of Barrett's dysplasia and early-stage esophageal adenocarcinoma, based on a Delphi process. Gastroenterology 2012;143:336-346.

4. Song EY, Frakes JM, Extermann M, et al. Clinical factors and outcomes of octogenarians receiving curative surgery for esophageal cancer. J Surg Res 2020;251:100-106.

5. Yibulayin W, Abulizi S, Lv H, Sun W. Minimally invasive oesophagectomy versus open esophagectomy for resectable esophageal cancer: a meta-analysis. World J Surg Oncol 2016;14:304.

6. Alvarez Herrero L, Pouw RE, van Vilsteren FG, et al. Risk of lymph node metastasis associated with deeper invasion by early adenocarcinoma of the esophagus and cardia: study based on endoscopic resection specimens. Endoscopy 2010;42:1030-1036.

7. Dunbar KB, Spechler SJ. The risk of lymph-node metastases in patients with high-grade dysplasia or intramucosal carcinoma in Barrett's esophagus: a systematic review. Am J Gastroenterol 2012;107:850-862.

8. Hirasawa K, Kokawa A, Oka H, et al. Superficial adenocarcinoma of the esophagogastric junction: long-term results of endoscopic submucosal dissection. Gastrointest Endosc 2010;72:960-966.

9. Manner H, May A, Pech O, et al. Early Barrett's carcinoma with "low-risk" submucosal invasion: long-term results of endoscopic resection with a curative intent. Am J Gastroenterol 2008;103:25892597.

10. Pimentel-Nunes P, Dinis-Ribeiro M, Ponchon T, et al. Endoscopic submucosal dissection: European Society of Gastrointestinal Endoscopy (ESGE) Guideline. Endoscopy 2015;47:829-854.

11. Wu J, Pan YM, Wang TT, Gao DJ, Hu B. Endotherapy versus surgery for early neoplasia in Barrett's esophagus: a meta-analysis. Gastrointest Endosc 2014;79:233-241.

12. Pouw RE, Seewald S, Gondrie JJ, et al. Stepwise radical endoscopic resection for eradication of Barrett's oesophagus with early neoplasia in a cohort of 169 patients. Gut 2010;59:1169-1177.

13. van Vilsteren FG, Pouw RE, Seewald S, et al. Stepwise radical endoscopic resection versus radiofrequency ablation for Barrett's oesophagus with high-grade dysplasia or early cancer: a multicentre randomised trial. Gut 2011;60:765-773.

14. Caillol F, Bories E, Pesenti C, et al. Radiofrequency ablation associated to mucosal resection in the oesophagus: experience in a single centre. Clin Res Hepatol Gastroenterol 2012;36:371-377.

15. Pech O, Behrens A, May A, et al. Long-term results and risk factor analysis for recurrence after curative endoscopic therapy in 349 patients with high-grade intraepithelial neoplasia and mucosal adenocarcinoma in Barrett's oesophagus. Gut 2008;57:1200-1206.

16. Caillol F, Godat S, Autret A, et al. Neoplastic Barrett's oesophagus and long-term follow-up after endoscopic therapy: complete histological eradication of Barrett associated with high-grade dysplasia significantly decreases neoplasia relapse. Surg Endosc 2016;30:5410-5418.

17. Haidry RJ, Butt MA, Dunn JM, et al; UK RFA Registry. Improvement over time in outcomes for patients undergoing endoscopic therapy for Barrett's oesophagus-related neoplasia: 6-year experience from the first 500 patients treated in the UK patient registry. Gut 2015;64:1192-1199.

18. Phoa KN, Pouw RE, Bisschops R, et al. Multimodality endoscopic 
eradication for neoplastic Barrett oesophagus: results of an European multicentre study (EURO-II). Gut 2016;65:555-562.

19. Anders M, Bähr C, El-Masry MA, et al. Long-term recurrence of neoplasia and Barrett's epithelium after complete endoscopic resection. Gut 2014;63:1535-1543.

20. de Matos MV, da Ponte-Neto AM, de Moura DTH, et al. Treatment of high-grade dysplasia and intramucosal carcinoma using radiofrequency ablation or endoscopic mucosal resection + radiofrequency ablation: Meta-analysis and systematic review. World J Gastrointest Endosc 2019;11:239-248.

21. Phoa KN, van Vilsteren FG, Weusten BL, et al. Radiofrequency ablation vs endoscopic surveillance for patients with Barrett esophagus and low-grade dysplasia: a randomized clinical trial. JAMA 2014;311:1209-1217.

22. Giovannini M, Bories E, Pesenti C, et al. Circumferential endoscopic mucosal resection in Barrett's esophagus with highgrade intraepithelial neoplasia or mucosal cancer. Preliminary results in 21 patients. Endoscopy 2004;36:782-787.

23. Lopes CV, Hela M, Pesenti C, et al. Circumferential endoscopic resection of Barrett's esophagus with high-grade dysplasia or early adenocarcinoma. Surg Endosc 2007;21:820-824.

24. Kuwano H, Nishimura Y, Oyama T, et al. Guidelines for diagnosis and treatment of carcinoma of the esophagus April 2012 edited by the Japan Esophageal Society. Esophagus 2014;12:1-30.

25. Pech O, Bollschweiler E, Manner H, Leers J, Ell C, Hölscher AH. Comparison between endoscopic and surgical resection of mucosal esophageal adenocarcinoma in Barrett's esophagus at two highvolume centers. Ann Surg 2011;254:67-72.

26. Pech O, May A, Manner H, et al. Long-term efficacy and safety of endoscopic resection for patients with mucosal adenocarcinoma of the esophagus. Gastroenterology 2014;146:652-660.

27. Weusten B, Bisschops R, Coron E, et al. Endoscopic management of Barrett's esophagus: European Society of Gastrointestinal Endoscopy (ESGE) Position Statement. Endoscopy 2017;49:191-198.

28. Conio M, Fisher DA, Blanchi S, Ruggeri C, Filiberti R, Siersema PD.
One-step circumferential endoscopic mucosal cap resection of Barrett's esophagus with early neoplasia. Clin Res Hepatol Gastroenterol 2014;38:81-91.

29. Yamasaki T, Hemond C, Eisa M, Ganocy S, Fass R. The changing epidemiology of gastroesophageal reflux disease: are patients getting younger? J Neurogastroenterol Motil 2018;24:559-569.

30. Coleman HG, Xie SH, Lagergren J. The epidemiology of esophageal adenocarcinoma. Gastroenterology 2018;154:390-405.

31. Qumseya B, Panossian AM, Rizk C, et al. Predictors of esophageal stricture formation post endoscopic mucosal resection. Clin Endosc 2014;47:155-161.

32. Li N, Pasricha S, Bulsiewicz WJ, et al. Effects of preceding endoscopic mucosal resection on the efficacy and safety of radiofrequency ablation for treatment of Barrett's esophagus: results from the United States Radiofrequency Ablation Registry. Dis Esophagus 2016;29:537-543.

33. Godat S, Caillol F, Autret A, et al. Endotherapy in case of relapse of neoplastic Barrett's esophagus after successful initial endoscopic resection. Surg Endosc 2017;31:3703-3710.

34. Caillol F, Godat S, Poizat F, et al. Probe confocal laser endomicroscopy in the therapeutic endoscopic management of Barrett's dysplasia. Ann Gastroenterol 2017;30:295-301.

35. Chennat J, Konda VJ, Ross AS, et al. Complete Barrett's eradication endoscopic mucosal resection: an effective treatment modality for high-grade dysplasia and intramucosal carcinoma--an American single-center experience. Am J Gastroenterol 2009;104: 2684-2692.

36. Moss A, Bourke MJ, Hourigan LF, et al. Endoscopic resection for Barrett's high-grade dysplasia and early esophageal adenocarcinoma: an essential staging procedure with long-term therapeutic benefit. Am J Gastroenterol 2010;105:1276-1283.

37. Agoston AT, Strauss AC, Dulai PS, et al. Predictors of treatment failure after radiofrequency ablation for intramucosal adenocarcinoma in Barrett esophagus: a multi-institutional retrospective cohort study. Am J Surg Pathol 2016;40:554-562. 